\title{
Ética de la reputación online, marca personal y privacidad en el cloud computing: protección de los usuarios frente al derecho al olvido
}

\author{
Pedro R. Palos-Sanchez \\ Rafael Robina Ramírez \\ Universidad de Extremadura - UEx, España
}

Luis Manuel Cerdá Suárez

Universidad Internacional de La Rioja - UNIR, España

ORIGINAL

\begin{abstract}
Resumen
Objetivo. Este artículo tiene como principal objetivo, analizar la situación actual y las previsiones futuras que puede tener la falta de privacidad en la nube o cloud computing. El borrado de los datos personales que indexa el buscador Google, retirar una foto de Facebook, eliminar un link de una noticia desafortunada o borrar el pasado puede ser una tarea que se nos antoje imposible. Aquellas personas afectadas por situaciones así, reclaman la aplicación del "derecho al olvido" basado en motivaciones plausibles.

Método. Este estudio ha contrastado las opiniones de afectados, abogados y expertos en reputación online y comunicación de marca personal.

Resultados. Los resultados demuestran la desproteción legal y la responsabilidad de los buscadores en la vulneración ética $y$, en algunos casos, legal de estos derechos.

Conclusiones. La privacidad va camino de convertirse en el principal quebradero de cabeza para los buscadores. Esta conclusión, no sólo proviene de las polémicas suscitadas, sino por las numerosas denuncias que señalan ahora a la herramienta y no sólo al origen de la información. Normalmente, la herramienta es el buscador, que pone al alcance general datos que los interesados preferirían suprimir o que permanezcan en el olvido.
\end{abstract}

\section{Palabras clave}

Cloud computing; Derecho al olvido; Ética; Reputación online; Transparencia; Tribunal de Justicia de la Unión Europea

\section{Ethics of online reputation, personal brand and privacy in cloud computing: protection of users against the right to forget}

\section{Abstract}

Objective. This article has as main objective to analyze the current situation and the future previsions that can have the lack of privacy in the cloud or cloud computing. Deleting personal data that indexes the Google binder, such as removing a photo from Facebook, deleting a link from an unfortunate news or erasing the past on the network can be a task that we feel impossible. Those who pursue objectives as diverse as those mentioned unanimously invoke the "right to forget" based on plausible motivations.

Method. This study has contrasted the views of stakeholders, lawyers and experts in online reputation and personal brand communication, to reach a series of conclusions of great utility for experts, managers and users in general.

Results. The results show the lack of legal protection and the responsibility of the search engines in the ethical and, in some cases, legal violation of these rights.

Conclusions. Privacy is going to become the main dilemma for search engines. This conclusion comes from the controversies and the numerous denunciations that point to both the origin of the information and the tool. Normally, the tool is the search engine, which provides general access to data that interested parties would prefer to delete or forget. 


\section{Introducción}

La evolución de la tecnología ha supuesto que en los últimos años Internet haya pasado de un medio de comunicación a la principal tecnología que soporta el cloud computing o, como se conoce en castellano, la nube. La existencia de la nube implica que cualquier proceso se realiza en una localización remota (de ahí el concepto nube), en lugar de en su ordenador personal, portátil o dispositivo móvil, aprovechando la potencia de computación a través de una conexión de internet. El concepto nube se utiliza, a menudo, para indicar un nuevo modelo o tecnología flexible, que proporciona recursos y servicios de Tl a través de Internet (Böhm et al., 2011).

Cloud Computing significa paradigma, porque los usuarios no necesitan conocimientos, experiencia o control sobre la propia tecnología (Wu et al., 2011). Se considera que la nube es un nuevo paradigma informático que permite a los consumidores utilizar temporalmente una infraestructura de red informática como un servicio proporcionado por proveedores externos (Youseff et al., 2008), donde se definen esas tecnologías como un servicio en el cual la empresa o el usuario paga sólo por el uso realizado (Palos y Correia, 2016a). La nube significa recibir multitud de servicios a través de internet de forma dinámica y escalable. Esta aceleración de la tecnología implica poder gestionar grandes cantidades de datos, informaciones y conocimientos, lo que a su vez lleva consigo importantes problemas de seguridad y privacidad (Gupta et al., 2013; Lian et al., 2014).

Hoy en día, empiezan a ser pocas las organizaciones que no tienen entre sus objetivos estratégicos realizar una correcta gestión del conocimiento que generan, de tal forma que este revierta en beneficios para la empresa (Palos y Aguayo-Camacho, 2016). Muchos directores de TI manifiestan esta preocupación (Sultán, 2011). Uno de los problemas que generan son la falta de privacidad y la vulneración al derecho a ser olvidado por hechos ocurridos en el pasado y que no se desea que sigan estando presentes en ficheros públicos, medios de comunicación u otros medios, como por ejemplo redes sociales. Esta vulneración afecta muy especialmente a la reputación online y a la propia marca personal, cuando el usuario afectado ejerce como profesional independiente. Estas cuestiones pueden tener origen en diferentes causas, como un error que se cometió en el pasado o, incluso, una falsedad vertida contra alguien, tergiversaciones de la realidad o simplemente informaciones privadas que aparecen.

\section{Antecedentes de la Privacidad y el Derecho al Olvido}

El concepto "derecho a olvidar" y el "derecho a ser olvidado" son términos diferentes. Mientras el primero se refiere un acontecimiento histórico no revitalizado debido al tiempo transcurrido hasta la fecha actual, el "derecho a ser olvidado" invoca el derecho de un individuo a remover ciertos datos de la red por diversas motivaciones (Mayer-Schönberger, 2009). Inicialmente el derecho al olvido puede ser considerado como contenido en el derecho de la personalidad, abarcando varios elementos como: la dignidad, el honor y el derecho a la vida privada. Las terminologías múltiples se utilizan en el contexto del derecho de la personalidad, principalmente el derecho a la integridad (moral y legal) de una persona que no debe ser infringida y para que una esfera de privacidad sea mantenida y distinguida.

Cuando abordamos la problemática asociada al "derecho al olvido", se debe, en primer lugar, distinguir distintos escenarios. El común denominador de estos diferentes escenarios es simpre el mismo: sucesos del pasado afectan a la reputación del ususario en el tempo presente y futuro. Los escenarios pueden ser diversos y tienen distinta legitimidad. Em este sentido hay escenarios clasificados como erróneos o por el contrario casuales. Otros afectan a la libertad del individuo, que se ve afectado por la libertad de opinión de los demás o que fruto del uso de su propia libertad ve que no hay olvido. En segundo lugar, es necesario clasificar los hechos entre aquellos que están dentro de lo que se llama derecho a la intimidad y los que están fuera de la privacidad.

Por un lado, el derecho a la intimidad, a mantener determinadas cosas en secreto, ya se ha extendido sin duda al derecho de los usuarios de Internet, protegiendo los datos privados en la red para decidir el posible uso que se hagan de dichos datos (Werro, 2009). Por otro, este derecho de privacidad podría impedir que los medios revelen ciertas verdades y hechos previamente divulgados solo si la información ya no es de interés público. Este no es el caso de aquella información que sigue siendo necesaria para proteger a los ciudadanos en los tiempos actuales. En este caso no se puede invocar el derecho al olvido (Werro y Belser, 1997). 
Especialmente común en la práctica judicial y de la doctrina jurídica ha sido el caso de personas condenadas en los tribunales por cometer determinados crímenes. En este caso, el derecho de privacidad se ampara en la irrelevancia informativa que para los ciudadanos tiene el saber que años anteriores un individuo pudo cometer algún delito. En este caso, los afectados, para poder comenzar una nueva vida solicitaron que esa información desapareciera, años después de haber cometido el delito (Weber, 2011).

Suiza es un buen ejemplo para el desarrollo del derecho a ser olvidado para evitar que, en el caso de los delincuentes el público siguiera teniendo acceso a los registros de aquellos durante una cantidad de tiempo sustancial (Swiss Federal Court, July 29, 1996). La práctica judicial reconoce así el derecho de un individuo a ser olvidado como un criminal (Swiss Federal Court, October 23, 2003).

En este caso, para los tribunales la discrecionalidad en la interpretación del término "cantidad de tiempo sustancial" transcurrido desde el inicio de una actividad delictiva es esencial. Por lo tanto, esos delitos: 1) Ya no son de interés público por lo que queda fuera de lugar cualquier intento por mantener en el recuerdo y, 2) Pueden ser contra producente para el objetivo de rehabilitar a la persona en cuestión.

Por lo tanto, el derecho al olvido se basa en la autonomía de un individuo que se convierte en titular de un derecho con respecto a la información personal en una escala de tiempo. Cuanto más se remonta el origen de la información, más probable es que los intereses personales prevalezcan sobre los intereses públicos (Weber, 2011).

Una vez esbozada una aproximación al concepto del "derecho al olvido" abordaremos otros aspectos derivados del gran crecimiento experimentado en el uso de la red y de la falta de una doctrina que lo regule. En el primer apartado justificaremos la necesidad de modernización de la legislación comunitaria actual, basada en la redefinición del concepto de "privacidad" que analizaremos en el segundo apartado, como límite para conservar un control efectivo sobre los datos personales. En el tercer apartado abordaremos la diferencia entre el derecho al olvido en Internet y el derecho al olvido que depende, exclusivamente, de los buscadores. En el cuarto apartado analizaremos el contenido de las críticas basadas en el no abuso de perfiles comerciales como uno de los modos de vehicular con contenidos difamatorios. En el quinto apartado abordaremos el origen del problema basado en la edición de páginas en Internet por el editor, en lugar del buscador. En el sexto apartado abordaremos la metodología del estudio y los resultados.

\section{Marco conceptual}

\subsection{El punto de partida: una legislación obsoleta}

Hoy en día, el mundo es una aldea global, donde es fácil que todos conozcamos, en cierta forma, la vida de los demás. El crecimiento exponencial del comercio electrónico ha significado que los consumidores generen comentarios, transacciones y experiencias de uso de los productos y servicios que se expresan a través de redes sociales. Todo esto influye en gran medida en otros consumidores (Brown y Reingen, 1987, Dellarocas, 2003, Godes y Mayzlin, 2004). El hecho de etiquetar una foto o divulgar una información que pertenece a la intimidad del individuo se convierte en un peligro potencial de naturaleza familiar o profesional, afectando no solo a la reputación online de un usuario sino también a la marca personal de un profesional que ejerce un trabajo por cuenta propia.

Este tipo de informaciones de carácter personal, que puede tener distintas procedencias en la red, es indexado por los buscadores al teclear nuestros nombre y apellidos, máxime si el individuo cuenta con apellidos poco comunes. Así, al alcance de un simple clic, pueden aparecer datos e informaciones de la vida del individuo que no guardan relación entre si, pero que dañan la reputación de este. Ejemplos como uma sanción administrativa, el sumario de un juicio donde simplemente se le cite como testigo, una multa de tráfico o una vinculación con hechos que provoquen alarma social, pueden ser resumidos de manera ordenada y oferecer una biografía atroz de la persona afectada. Todo ello puede ser una fuente de problemas personales con incidencias profesionales. De este modo, los buscadores, webs o blog que simulan ser medios digitales o redes sociales mantienen en tempo presente aquello que pertenece a un pasado que en el entorno digital daña la reputación del individuo. Se 
trata en estos casos del problema de la ausencia de temporalidad. Esta ausencia de temporalidad hace que los hechos tengan aún más credibilidad, dañando la reputación.

Hasta hace muy poco tiempo, la normativa europea que estaba vigente era del año 1995 (1995/46/CE1) y ha sido revisada recientemente por la Comisión Europea. Esta remitió al Parlamento y otras instituciones europeas un documento de reforma, que ha permitido actualizar la legislación en materia de protección de datos.

Esta Directiva sobre protección de datos (1995/46/CE1) contaba con déficits relevantes que debían ser actualizados, en gran parte por la eclosión de las redes sociales. Fundamentalmente, carecía de aspectos que protegieran adecuadamente derecho a la intimidad con respecto al tratamiento de datos personales, así como otros derechos a oponerse al tratamiento de los datos personales en determinadas situaciones.

Así, el pasado 4 de mayo de 2016, se publicó el Reglamento (UE) 2016/679 del Parlamento Europeo y del Consejo, de 27 de abril de 2016, relativo a la protección de las personas físicas en lo que respecta al tratamiento de datos personales y a la libre circulación de estos datos. Por tanto, si bien el Reglamento entró en vigor el 24 de mayo de 2016, no será de aplicación hasta el 25 de mayo de 2018, momento en el que quedará derogada la Directiva 95/46/CE.

A ello, ha contribuido el uso del cloud computing, ya que su uso afecta especialmente a la internacionalización de los datos, ya que algunos centros que dan soporte con servidores están radicados en países sin legislación de protección de datos. A todo esto, debemos añadir que en territorio de la Unión Europea la legislación sobre privacidad es diferente. Por tanto, pareció muy necesario que la Comisión adoptara un instrumento de aplicación a todo el territorio europeo por igual. Sin embargo, el caso de países de fuera de la UE no se puede contemplar.

El nuevo reglamento aprobado, establece en su capítulo III (artículos 12 a 21) un desarrollo de los derechos de los interesados. Con esto se pretende que la normativa nacional de los países miembros de la UE, en materia de protección de datos, introduzcan nuevos derechos. Entre estos derechos estarían los de supresión o derecho al olvido y, también, aquellos relacionados con portabilidad de datos. Entre los primeros, estarían los denominados derechos ARCO (acceso, rectificación, cancelación y oposición). Entre los segundos, aquellos relacionados con la portabilidad de los datos en diferentes ámbitos, como por ejemplo cuando cambiamos de proveedor en la nube.

El nuevo reglamento establece claramente derechos relacionados con la Transparencia (art. 12); Información de los datos (arts. 13 a 14); Acceso a los ficheros (art. 15); Rectificación de los datos que contengan información del ciudadano (Art. 16); la Supresión o Derecho al Olvido (art. 17) que es el tema central de esta investigación y además: de la Limitación del tratamiento de los datos (art. 18); de la Portabilidad de datos (art. 20) y de la Oposición (art. 21).

Respecto a la Portabilidad, debemos indicar que cuando un ciudadano proporciona datos, el titular del fichero automatizado debe poder proporcionar esos datos para poder permitir el cambio de un servicio. Y esto, siempre se debe hacer en un formato que permita esa misma portabilidad a otro responsable. Este nuevo derecho a la portabilidad está condicionado por el estado de la tecnología, pero se interpreta que se debe respetar el derecho al olvido, excepto si es de interés público o en el ejercicio de administraciones públicas conferidos al responsable del tratamiento.

\subsection{De la protección a la transparencia de los datos}

La privacidad va camino de convertirse en el principal quebradero de cabeza para los buscadores. Este aseguramiento que establece el derecho a la portabilidad garantiza el trasvase de datos en territorio de la UE. Pero la cuestión, estriba también en asegurar la privacidad del usuario. Es decir, la recogida de datos del usuario debe garantizar el consentimiento de este. Para ello, direcciones IP, "huellas digitales" o metadatos deben ser tratados como tales porque forman parte de la privacidad del usuario. Así, el papel de los operadores tecnológicos empieza a estar en cuestión. En concreto de operadores de redes sociales, de buscadores o de mensajería. En concreto y de manera muy especial, el buscador facilita que no se ejerza el derecho al olvido, ya que pone al alcance general datos que los interesados preferirían sepultar en el olvido (Saura, Palos y Debasa, 2017). 
Los últimos problemas de Google en relación con la privacidad -la captación de datos sin autorización a través de redes Wi-Fi públicas no han hecho sino amplificar una percepción que ya estaba muy extendida: internet es un auténtico agujero de falta de privacidad, por donde circulan todo tipo de datos personales, en ocasiones sin conocimiento de los interesados.

Otros casos similares lo podemos encontrar en Facebook. El avance de las redes sociales ha sido muy importante y alertan de estos riesgos. En este caso, Facebook cambió en varias ocasiones la política de privacidad pudiendo demostrar con ello su vulnerabilidad en su capacidad de proteger los datos de los usuarios.

En el caso de los buscadores, el propósito del almacenamiento de los datos es la disponibilidad para otros usuarios, pero parece que la continuada multiplicación de denuncias contra los buscadores demuestra que esto no parece legal. Esto aumenta la falta de confianza en la publicación de muchos datos personales por la falta de rigor que los propios buscadores tienen a la hora de distinguir en la licitud de la propia información que indexa.

Muchas denuncias de usuarios se refieren a hechos como el impedimento de redes sociales para recuperar datos personales, como fotos personales, con lo que se ha limitado su derecho al acceso, rectificación, bloqueo o supresión de datos propios. En el artículo 17 del nuevo reglamento se reconoce expresamente un nuevo derecho, el derecho de supresión, más comúnmente conocido como "derecho al olvido". Como ya hemos comentado anteriormente, este derecho ya había sido objeto de reconocimiento por la jurisprudencia. El derecho al olvido, conocido como de supresión, se refiere a la garantía del usuario frente a los titulares de los datos a obtener, sin dilación indebida, la supresión de los datos personales que le conciernan del responsable del tratamiento en determinados supuestos, entre otros, cuando los datos no sean necesarios para las finalidades para las que fueron recogidos, cuando los datos personales hayan sido tratados ilícitamente o cuando los datos personales deban suprimirse para cumplir con una obligación legal establecida en la legislación aplicable al responsable del tratamiento (Mayor, 2016).

El nuevo reglamento establece que el responsable que esté obligado a suprimir datos personales deberá adoptar medidas razonables, teniendo en cuenta la tecnología disponible y el coste de su aplicación, así como informar a los responsables que estén tratando los datos personales de la solicitud del interesado de supresión de cualquier enlace a esos datos personales, o cualquier copia o réplica de estos.

Ahora bien, existen algunas excepciones al ejercicio del derecho de supresión (Mayor, 2016), como para ejercer el derecho a la libertad de expresión e información, que se refiere principalmente a medios de comunicación, webs o redes sociales; para el cumplimiento de una obligación legal que requiera el tratamiento de datos impuesta por la legislación aplicable al responsable del tratamiento, o para el cumplimiento de una misión realizada en interés público o en el ejercicio de poderes públicos conferidos al responsable.

a) Por razones de interés público en el ámbito de la salud pública.

b) Con fines de archivo en interés público, fines de investigación científica o histórica o fines estadísticos, en la medida en que el ejercicio del derecho de supresión pudiera hacer imposible u obstaculizar gravemente el logro de los objetivos de dicho tratamiento.

c) Para la formulación, el ejercicio o la defensa de reclamaciones.

\subsection{El derecho al olvido en Internet y los buscadores}

El Tribunal de Justicia de la Unión Europea dictaminó que los motores de búsqueda o buscadores estarán obligados a desindexar enlaces a datos publicados en el pasado, si estos son lesivos para el individuo y, además, no tienen la consideración de relevancia pública. Esta relevancia viene por la naturaleza de la actividad del individuo. Dicha sentencia se basa en el carácter personal de los datos, estableciendo que toda la normativa existente también es aplicable a los enlaces que proporcionan los motores de búsqueda.

Ahora bien, esta sentencia contradice la posición de la compañía predominante en el sector de buscadores. Google ha defendido la no aplicación de esta doctrina por ser una compañía norteamericana. Este fallo y la regulación aprobada en el Parlamento Europeo dejan al descubierto que no es una práctica legal ignorar la 
normativa por parte del buscador. Sin embargo, la Unión Europea precisa que todos los países miembros adopten en un periodo transitorio esta normativa, que España ha incorporado a la LOPD (Ley Orgánica de Protección de Datos). No obstante, todo esto es sólo y exclusivamente de aplicación a las personas físicas, no a empresas, marcas, organizaciones o sociedades.

Por tanto, el fallo del Tribunal y la normativa aprobada, indica que es totalmente factible que un buscador atienda la demanda de una persona de quitar un vínculo a noticias o informaciones que atenten contra el derecho al olvido, con independencia de que estos sean ciertos. La sentencia indica la prevalencia de la protección de los datos personales frente a los intereses económicos de la compañía que ofrece el servicio del motor de búsquedas. No obstante, la sentencia no entra en llegar al fondo de la cuestión, es decir el derecho a que el editor de la información la retire de la red. De ahí, la importancia de distinguir claramente entre motor de búsqueda y editor.

Por último, para cerrar esta exposición de la situación actual, debemos indicar que el individuo afectado debe dirigirse directamente a la compañía responsable del buscador y exponer los enlaces, así como los motivos que de manera razonada indican que deben desindexar de las búsquedas por razones de derecho al olvido, de falsedad o de que atentan contra su privacidad. Será la compañía responsable quién lo decida en base a la veracidad, el tiempo transcurrido y a la exposición pública del afectado. Si la compañía del buscador deniega desindexar el o los enlaces, el individuo que se ve afectado podrá dirigirse a la autoridad responsable de la protección de datos.

Por tanto, la situación actual deja en las circunstancias del caso concreto el cumplimiento de ese derecho al olvido, que en muchos casos se escuda en la relevancia pública del afectado para no retirar los enlaces. En otros, el tiempo que transcurre hace que se disemine por toda la red una información que de haber actuado con rapidez y diligencia no hubiese lesionado los derechos del afectado. Es decir, es tarde para eliminar todos los enlaces porque se eliminan unos y aparecen nuevos, a veces fruto de la "viralidad" con que se propagan las informaciones en la red y en otros casos de la propia dificultad técnica.

\subsection{Del buscador al editor: la búsqueda del origen del problema}

La posición de dominio de Google en el sector de motores de búsqueda es de absoluta hegemonía. Para los usuarios es absolutamente fiable y el hábito de uso lo convierte en un producto irrenunciable para el usuario (Palos y Saura, 2018). Por tanto, tal vez el debate de la regulación de los buscadores no es desacertado. La fiabilidad y equidad en la presentación de los enlaces de búsqueda pasan inadvertidos para los usuarios, que aún quedamos fascinados por su utilidad y facilidad de uso. Pero detrás, se esconden derechos vulnerados, como en el caso que nos ocupa o informaciones falsas que no se desindexan. Además, muchos usuarios creen todo lo que presenta el buscador sin dudar de su veracidad, como si el buscador fuera una agencia de noticias o un medio de comunicación que contrasta el origen de la información. No debemos olvidar, que resulta más fácil y económico desindexar que llegar hasta el editor de la información. Por lo tanto, parece importante pensar en la regulación del papel de los motores de búsqueda.

\subsection{El crítico sistema de intercambio de información personal: El caso de Google}

El nuevo sistema de intercambio de información personal es muy crítico en el caso de Google, debido a que unifica y los trata como de un usuario único. De facto, esto significa intercambiar información personal entre Gmail, YouTube, Google+ y otros. Este nuevo sistema hace que el buscador sea crítico a la hora de cumplir el derecho al olvido, ya que disemina toda la información por todos sus servicios, que por otro lado gozan de una popularidad casi unánime. Tampoco está claro que la defensa de la privacidad garantice un "derecho al olvido" cuando se trata de información pública.

Los tres aspectos que hacen que Google se vea afectada por un aluvión de críticas son: 1) Ofrecer sin consentimiento del usuario, datos que permiten desarrollar perfiles comerciales; 2) pone a disposición de los usuarios contenidos, con independencia de que estos sean difamatórios o no; 3) por último, en algunos casos no desindexa las informaciones, a pesar de que estas sean antiguas o, en ciertos casos, falsas.

Aunque el documento de la Comisión Europea aborda el tema de la protección de datos desde una perspectiva general, es significativo que ya en el tercer párrafo aparezcan mencionadas las redes sociales. La comunicación también menciona explícitamente el otro gran objeto de las últimas polémicas: la publicidad relacionada con el 
comportamiento (behavioural advertising). Este tipo de publicidad analiza el comportamiento del usuario en Internet para componer un perfil comercial personalizado y remitirle los anuncios que cuadren con ese perfil. Los consumidores forman parte de la información creada por los usuarios de sitios web que ya han comprado el producto de destino.

Estos contienen información y recomendaciones de los productos desde la perspectiva del consumidor (Park et al., 2007). Estudios recientes han investigado como el comercio electrónico influye en el comportamiento del consumidor (Brown y Reingen, 1987; Chatterjee, 2001; Chen y Xie, 2008). Por ejemplo, las personas toman decisiones de compra basadas en información creada por el consumidor a través de Internet (Godes y Mayzlin, 2004) y se basan en anuncios en Internet para tomar decisiones que van desde qué película mirar a qué acciones invertir (Dellarocas, 2003).

El problema es cómo se realiza la radiografía comercial del usuario. Google tuvo que afrontar una fuerte polémica porque utilizaba los contenidos de los correos de Gmail para generar los anuncios más adecuados. Facebook, por su parte, ofrece a sus usuarios valorar los anuncios que coloca en las cuentas personales, de tal modo que a través de esas valoraciones -me interesa, no me interesa, no volver a mostrar, etc.- se va creando un perfil suculento para las empresas, que así pueden llegar de manera más directa y efectiva a su público objetivo.

Las compañías deberían pedir permiso al usuario para utilizar sus datos con un fin distinto de aquel para el que se solicitaron. Para algunas asociaciones de usuarios estas prácticas suponen una vigilancia del usuario con fines estrictamente comerciales, y en algunos casos vulneran directamente la privacidad del internauta, como por ejemplo al recoger datos de los correos electrónicos sin un aviso previo y explícito.

\section{Metodologia}

Después de revisar la literatura, se realizó un estudio cualitativo con el fin de probar y ajustar el marco conceptual. Este estudio cualitativo buscó una forma más cómoda y relacional, en la que distintos profesionales y personas afectadas se sintieran cómodas hablando sobre el tema de estudio.

El objetivo perseguido fue, por tanto, realizado desde una manera espontánea y natural, indicando qué factores determinan la falta de ética en los buscadores, medios de comunicación y foros, donde la protección de los usuarios frente al Derecho al Olvido no se viene cumpliendo en muchos casos.

La aplicación de esta metodología cualitativa, materializada a través de la entrevista en profundidad, ha supuesto para los investigadores, contar con un componente cualitativo de extraordinaria importancia para la investigación.

\subsection{La metodología cualitativa}

La literatura identificó tres perspectivas teóricas en el estudio de las ciencias sociales (Coutinho, 2008): la positivista que busca conocer los hechos y las causas de los fenómenos sociales con independencia de los estados subjetivos de los sujetos, fenomenológica que busca comprender los fenómenos sociales desde el punto de vista o perspectiva de los autores y la crítica o sociocrática: no sólo la simple combinación de métodos sirve para conseguir los objetivos que se persiguen.

El enfoque cualitativo se ve favorecido en el proceso de diseño teórico (Punch, 1998). Los números dependen de los significados y los significados también dependen de la cantidad y la cuantificación, incluso en el campo de la descripción numérica meros casos, son un apoyo importante para los argumentos.

Como Glaser y Strauss (1967) identificó, aplicando la teoría enraizada, ambas formas de recogida y tratamiento de los datos son necesarios, al no ser la cantidad, más que un simple método de prueba cualitativa se debe utilizar en forma de suplemento de verificación mutua y, lo más importante, que al comparar ambos producen teoría.

Como se ha señalado por estos autores, los métodos cualitativos y cuantitativos se pueden combinar con el fin de llegar a ser la metodología más completa en un estudio empírico. Siguiendo esta línea de pensamiento, este trabajo de investigación supone el punto de partido de otro posterior y una aproximación a un futuro trabajo de naturaleza cuantitativa. 
A partir de un estudio de carácter cualitativo se pueden identificar algunos de los factores importantes para el ajuste de las construcciones de un estudio cuantitativo.

\subsection{Metodología de recogida de datos}

Se utilizaron entrevistas semiestructuradas, en la que se evaluó la capacidad de lectura del modelo de enfrentar la realidad estudiando y esperar es una colección de datos en favor del proceso iterativo característica de la construcción teórica de las metodologías adoptadas.

Todas las entrevistas duraron entre 15 y 30 minutos y se llevaron a cabo personalmente por los autores de este trabajo. Se definieron los objetivos y temas de discusión con el fin de mejorar la información relevante para la reevaluación del marco conceptual, basado en las percepciones de los actores. Todas las preguntas fueron diseñadas con el fin de permitir a los entrevistados hablar en general de los temas propuestos.

La Tabla 1 muestra el marco conceptual adoptado.

Tabla 1. Conceptos, dimensiones e indicadores propuestos

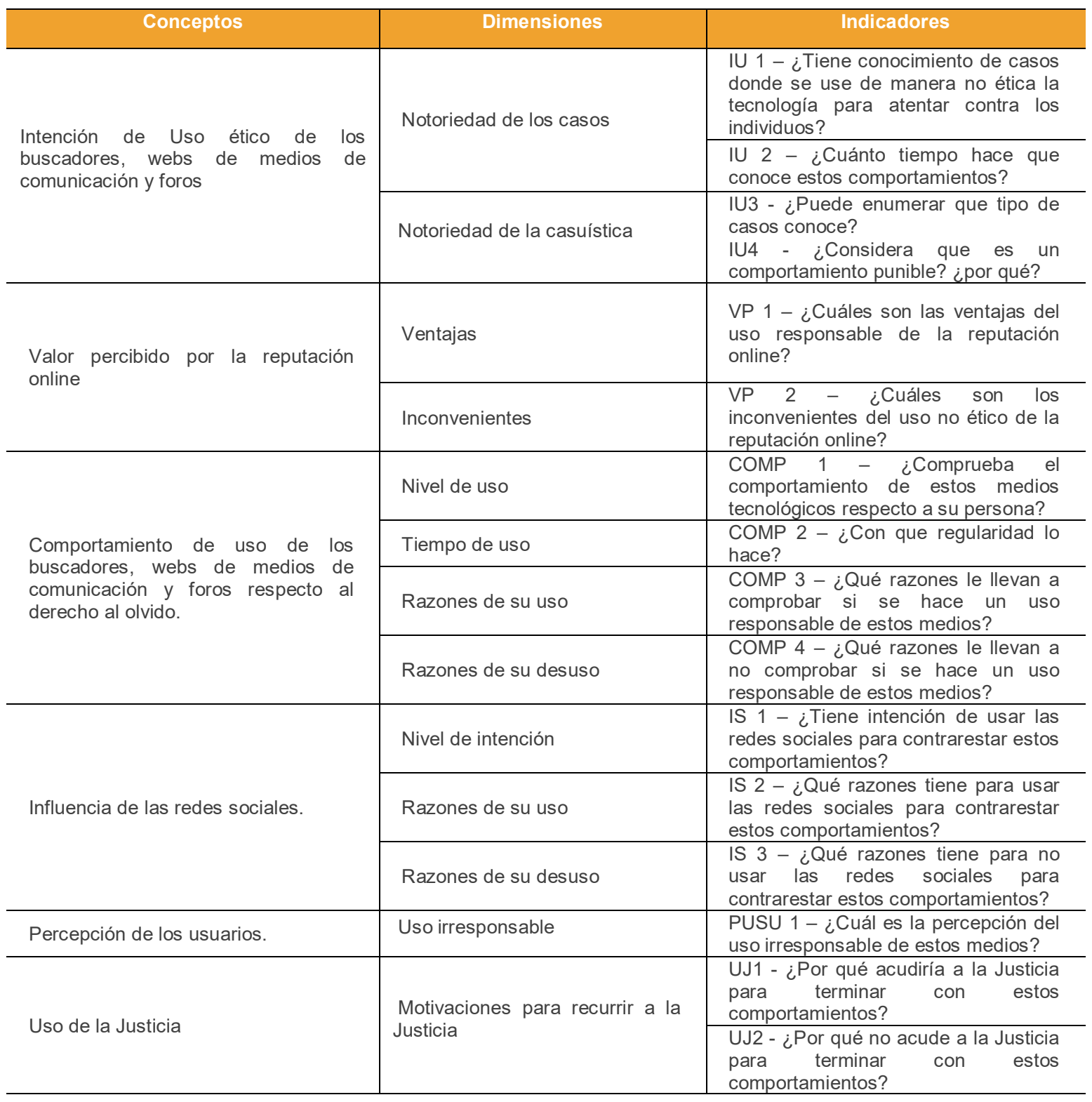




\subsection{Muestra}

El muestreo es una técnica que permite el conocimiento científico de la realidad y se debe utilizar cuando otros métodos alternativos, por diversas razones, no se muestran adecuados por coste o tiempo, o pudieran ser posibles. La elección del muestreo se aplicó recurriendo a técnicas no probabilísticas o dirigidas por los investigadores. El tipo de estudio y el tamaño de su universo contribuyeron a esta definición. El tamaño de la muestra fue de 25 entrevistas.

La elección del muestreo se llevó a cabo con técnicas no probabilísticas, ya que el tipo de estudio y el tamaño de su universo contribuyeron a esta definición.

La elección de los elementos de la muestra se realizó mediante los criterios de accesibilidad de los encuestados, si bien hubo recomendaciones por los entrevistados inicialmente hacia nuevas entrevistas. De esta forma, combinando un método de recomendación con otro de conveniencia, se alcanzó la muestra.

Así las primeras cinco entrevistas se consideraron como de instrucción; se evaluaron las reacciones de los que respondieron, respecto a la duración, el tipo de cuestiones y temas tratados. Se detectaron algunos problemas específicos después de la finalización de la entrevista, asegurando que el guion propuesto originalmente era adecuado respecto a la investigación que se pretendía realizar.

La población entrevistada consiste en clientes actuales de despachos de abogados y agencias de comunicación preocupados por su reputación online, con problemas derivados de investigaciones judiciales, donde la política tiene alguna influencia, despidos, embargos o casos sobreseídos cuyo resultado final no se publican posteriormente o simplemente absueltos tras la fase de instrucción o tras recurso a instancia superior. Además de los clientes, se entrevistaron en menor proporción a responsables de agencias de comunicación digital y abogados expertos en derecho al olvido.

\section{Análisis de los resultados}

El análisis de las entrevistas produjo resultados importantes para la confirmación de un modelo teórico a priori y también contribuyó con unas dimensiones no consideradas anteriormente.

Los resultados del análisis de los comportamientos de uso fueron de gran interés, así como algunas de las dimensiones de actitud, las cuales resultaron importantes.

\subsection{Intención del uso ético de los buscadores, webs de medios de comunicación y foros.}

\subsubsection{Notoriedad de los casos}

El conocimiento de casos donde se use de manera no ética la tecnología para atentar contra los individuos ha resultado ser del $93 \%$, casi todos los entrevistados conocen algún caso similar al suyo o trabajan en alguno.

Respecto al tiempo que conocen estos comportamientos, la media obtenida supera los 5 años y coincide con el uso masivo de los medios digitales y su uso en la prensa escrita. Otro detalle coincidente es la creciente judicialización de la vida pública en España.

\subsubsection{Notoriedad de la casuística}

Todos los entrevistados pudieron enumerar los casos que conoce y consideraron el comportamiento punible el $80 \%$. Las razones fueron diversas desde filtraciones de funcionarios del Ministerio del Interior a periodistas, lo que atenta contra la Ley de Enjuiciamiento Criminal, hasta intencionalidad política o empresarial del propio periodista o usuario del foro o red social donde aparece el comentario.

A continuación, se presenta un ejemplo de respuestas para cada una de las categorías más representativas: 
Entrevistado $n^{\circ}$ 5: "Conozco casos de personas imputadas que, durante la investigación judicial, el fiscal sobreseyó su caso, ningún medio lo publicó posteriormente y la noticia sigue publicada a pesar de ello".

Hombre, de 55 años, directivo empresarial, 250 empleados a su cargo en la actualidad.

Entrevistado $n^{\circ}$ 3: "En mi caso la noticia apareció 12 horas antes de que se produjese un acontecimiento similar al que se publicó y que Google ni siquiera retira, pese a reiteradas solicitudes".

Hombre, de 42 años, profesional autónomo.

\subsection{Valor percibido por la reputación online}

\subsubsection{Ventajas}

Las ventajas del uso responsable de la reputación online se destacan fundamentalmente en que constituye un refuerzo positivo en la intención de compra e incluso de estímulo, ya que genera seguridad en la decisión y percepciones positivas durante la prestación de los servicios contratados. En especial, genera seguridad en el desarrollo de las actividades cuando estas son contratadas. A personas que no desarrollen actividades de naturaleza mercantil, les afecta en su autoestima y seguridad personal.

En cuanto a los inconvenientes de la reputación online, su afección en sentido negativo perjudica a la confianza que se deposite en la empresa. Especialmente, afecta a los profesionales especializados que ejercen su actividad en sectores como Sector Público, Salud, Ingeniería, Arquitectura o Derecho. La confianza está estrechamente relacionada con la intención de compra o de contratar determinados servicios, en especial profesionales.

Entrevistada $n^{\circ} 1$ - "Trabajo con casos que van desde políticos hasta tiendas y restaurantes. En todos los casos, la persona afectada muestra síntomas de nerviosismo e inseguridad por perder seguridad en lo que hace y temer lo que le puedan reportar estos mensajes negativos en su futuro".

Mujer, de 55 años, publicista y community manager.

\subsection{Comportamiento de uso de los buscadores, webs de medios de comunicación y foros respecto al derecho al olvido.}

\subsubsection{Nivel de uso}

La mayoría de los entrevistados comprueban si los medios, foros y buscadores continúan teniendo publicado esos mensajes que afectan a su reputación online.

\subsubsection{Tiempo de uso}

La mayoría de los entrevistados comprueban con una regularidad que va de quincenal a mensual la retirada de los mensajes o las comunicaciones negativas. En algún caso, incluso semanal.

Entrevistado $n^{\circ}$ 4: "Recibo diariamente de lunes a viernes, un informe de prensa sobre noticias relacionadas con aquellas que afectan a mi reputación online, ya que es un asunto colectivo que afecta a personas en mi situación".

\subsubsection{Razones de su uso}

Los entrevistados comprueban si se hace un uso responsable de estos medios porque están pasando situaciones que no desean a sus semejantes. Están fuertemente solidarizados con situaciones similares.

Entrevistado $n^{\circ}$ 14: "Estas situaciones pueden llegar a afectar con cuadros psicológicos como la depresión, el trastorno de ansiedad o el suicidio. Existen docenas de casos documentados en España que han acabado en quitarse la vida. El uso desproporcionado con fines instrumentales y partidistas, por parte de los medios ha provocado episodios que influyen en trágicos desenlaces, como el de la Senadora española Rita Barberá fallecida recientemente". 
Hombre de 62 años, funcionario, padre de 3 hijos y abuelo de 7 nietos.

\subsubsection{Razones de su desuso}

Las razones que han expresado los entrevistados, que le llevan a no comprobar si se hace un uso responsable de estos medios es sobre todo la práctica del olvido, como terapia para superar episodios traumáticos personales o profesionales. En algunos casos, rehacer su vida o dejar en manos de profesionales de la Comunicación o del Derecho la resolución de su caso, ante la frustración que produce este vacío legal.

Entrevistado $n^{\circ}$ 8: "La llamada pena de telediario constituirá una salvajada moral para generaciones futuras."

Hombre, 51 años, Comercial, eximputado y sobreseído por un Fiscal en la fase de Instrucción judicial.

Entrevistado $n^{\circ}$ 10: "La justicia parece que se imparte de manera preventiva haciendo que los medios publiquen imputaciones que muchas vecen sólo tienen un fin preventivo para la sociedad de nuestro tiempo. ¿Es eso justo?".

Mujer de 33 años, Abogada y madre de familia. Letrada en un caso donde hay un menor afectado.

Entrevistado $n^{\circ}$ 13: "La presunción de inocencia no existe en Internet y eso es muy muy grave en un Estado de Derecho".

Hombre, 52 años, Profesor de Educación Infantil, más de 25 años impartiendo enseñanza.

Entrevistado $n^{\circ}$ 14: "Medios de comunicación, en especial digitales, aprovechan los buscadores para matar públicamente la reputación de aquellas empresas y profesionales que afecten o puedan llegar a afectar a los intereses que representan".

Hombre, Licenciado en Filosofía y Letras, Exalcalde y Parlamentario Autonómico.

\subsection{Influencia de las redes sociales.}

\subsubsection{Nivel de intención}

Respecto a la intención de usar las redes sociales para contrarrestar estos comportamientos, un $60 \%$ de ellos afirma haberlo hecho. De igual forma, usan las redes sociales con otros fines, como estar en contacto con amigos y conocidos. Aquellos entrevistados que no usan habitualmente las redes sociales, tampoco han visto en ellas la forma de contrarrestar o prodigar mensajes que mejoren su reputación online.

\subsubsection{Razones de su uso}

Los entrevistados han encontrado en un 33\% de los casos mensajes que han podido dar a conocer los mensajes negativos, para contrarrestarlos con pruebas o alegaciones como el tiempo transcurrido. No hay un consenso en cuanto es ese tiempo.

Entrevistado $n^{\circ}$ 2: "Las razones del embargo de mi casa nunca se publicaron. Sólo en las redes sociales lo he contado".

Hombre 25 años, Expropietario de una vivienda.

\subsubsection{Razones de su desuso}

Aquellos entrevistados que no usan las redes sociales, no lo hacen porque confiesan desconocer su uso o porque no son verdaderamente conscientes del resultado que esto pueda tener Ante la duda, prefieren no usar las redes y no dar más protagonismo a los mensajes o simplemente a su persona. 
Entrevistado $n^{\circ}$ 5: "La sociedad actual impulsa a los individuos con algún tipo de relevancia pública, aunque sea mínima, a mantener un perfil bajo en redes sociales".

Hombre, 42 años, Experto en Comunicación y Marketing Digital.

\subsubsection{Percepción de los usuarios}

La percepción que los entrevistados creen que tienen estas noticias es mucho más baja de la que inicialmente creían. Sólo aquellas personas cercanas a los afectados le han dado más importancia a la misma.

\subsubsection{Uso de la Justicia}

Tan sólo un $20 \%$ de los entrevistados han decidido formalmente pasar a la acción e interponer demandas contra el honor o ejercer el derecho al olvido contra medios o buscadores. El resto desconoce como el Derecho les puede asistir o prefieren no incurrir en gastos procesales. Tan sólo 2 casos entrevistados no creen que la Justicia pueda solucionar su situación.

\section{Conclusiones}

El derecho a ser olvidado, como derecho fundamental, ha cobrado en los últimos años una importancia especial, debido a dos razones principalmente: 1) Por la permanente digitalización de la vida personal y profesional de las personas que permite tener un fácil acceso a todo tipo de información (Brown y Reingen, 1987, Dellarocas, 2003, Godes y Mayzlin, 2004). 2) Por la necesidad defensa de la intimidad personal protegiendo los datos privados en la red para decidir el posible uso que se hagan de dichos datos (Werro, 2009). Entre las limitaciones de este derecho están que sólo afecta a las personas físicas y que puede entrar en conflicto con otros derechos fundamentales (libertad de información y de expresión). En este último caso, el primero o el segundo cede ante el otro, dependiendo de las circunstancias.

Pero la privacidad necesaria a la que todo individuo tiene derecho puede chocar la libertad de los medios para revelar ciertas verdades. Existe una colisión de unos derechos personales contra otros, es necesario remarcar que la libertad de prensa entra en conflicto con la protección de los derechos personales, por lo que se recomienda considerar los criterios ya mencionados -los del interés periodístico de los datos judiciales, de interés histórico y de interés público por el desempeño de los funcionarios- para resolver si es apropiado o no mantener la información presente en Internet o proceder a la eliminación de dichos datos de la Web (Silberleib, 2016).

En ocasiones, el derecho al olvido se contempla si, una vez revelada esa información, pasado un tiempo razonable, los hechos divulgados dejan de tener interés público. En este caso, no se puede invocar el derecho al olvido (Werro y Belser, 1997), al tratarse de personas condenadas en los tribunales por cometer determinados crímenes. Cuanto más se remonta el origen de la información, más probable es que los intereses personales prevalezcan sobre los intereses públicos (Weber, 2011). Así viene expresamente recogido en una sentencia del Tribunal de la Justicia de la Unión Europea. Dicha institución ha dictaminado que los buscadores de Internet están obligados a retirar los enlaces a informaciones publicadas en el pasado, si resultan lesivas para una persona y carecen ya de relevancia pública.

La privacidad va camino de convertirse en el principal quebradero de cabeza para los buscadores. Esta conclusión, no sólo proviene de las polémicas suscitadas, sino por las numerosas denuncias que señalan ahora a la herramienta y no sólo al origen de la información. Normalmente, la herramienta es el buscador, que pone al alcance general datos que los interesados preferirían suprimir o que permanezcan en el olvido.

Por otro lado, el vertiginoso avance de las redes sociales ha provocado nuevos problemas relacionados con la necesaria privacidad del usuario. Estos no están relacionados directamente con el "olvido de datos" sino con el uso de datos de manera fraudulenta para fines comerciales. Es decir, los datos son almacenados con el propósito de atender al interés de las empresas comerciales. De este modo, no es infrecuente recibir anuncios de empresas comerciales cuando navegas por internet estrechamente relacionada con búsquedas anteriores.

Es por ello por lo que la Comisión Europea ha garantizado la confianza del usuario en la red, sino también para promover la transparencia por parte del responsable del tratamiento de los datos (Palos y Correia, 2016b). Y 
para ello, em el artículo 17 del nuevo reglamento se reconoce expresamente un nuevo derecho: el derecho de supresión o "derecho al olvido" que se refiere a la garantía del usuario frente a los titulares de los datos a obtener, sin dilación indebida, la supresión de los datos personales que le conciernan del responsable del tratamiento en determinados supuestos, entre otros, cuando los datos no sean necesarios para las finalidades para las que fueron recogidos, cuando los datos personales hayan sido tratados ilícitamente o cuando los datos personales deban suprimirse para cumplir con una obligación legal establecida en la legislación aplicable al responsable del tratamiento.

Por tanto, en aras del mantenimiento de aquella confianza, ha sido especialmente importante el fallo del Tribunal por la que en ningún caso prevalecerá el interés económico del buscador frente a la protección de datos. Así, la sentencia añade que los particulares podrán ejercer el derecho al olvido frente a los buscadores, consagrando así el llamado "derecho al olvido" para que los datos lesivos -aunque sean ciertos- no queden para siempre accesibles en Internet.

Aunque el Tribunal no entra en si también podemos ejercerlo frente a los editores de la información. De este modo no reconoce un derecho absoluto al olvido, es decir, que desaparezca dicha información de internet del soporte original en que se publicó. En este caso, habrá que habrá que buscar un equilibrio entre la privacidad y libertad o derecho a la información, ponderando en cada caso lo que es más adecuado.

Por último, debemos reflexionar colectivamente sobre la posición dominante que tiene el motor de búsqueda Google a la hora de decidir unilateralmente que indexa y durante cuánto tiempo. Datos publicados el año pasado (Statista, 2015), señalan que Google abarca el 89\% de las búsquedas mundiales; Yahoo 3\%; Bing 3\% y Baidu $3 \%$ (el «Google chino») y, otros, el resto de los buscadores porcentajes no significativos. Esta posición de práctico monopolio pone en jaque la propia libertad de expresión, que en muchos casos queda reducida a lo que el buscador crea que deba ser indexado y por tanto localizado. Es como si pudiéramos expresarnos, pero sólo "oímos" aquello que Google desea.

Ya son casi 5 años los que lleva investigando la Comisión Europea (CE) a Google. De hecho, existen ya acusaciones formales por abuso de su posición de dominio, en concreto por favorecer sus propios productos en las búsquedas en Internet (Google Shopping) en las búsquedas de productos en venta online. De la misma forma, también Google obligó a los fabricantes de Smartphone a usar su sistema operativo para móviles: Android.

En la UE existen unas normas, y estas no pueden obstaculizar el desarrollo y el acceso al mercado de sistemas de explotación, aplicaciones y servicios para dispositivos móviles inteligentes rivales de Android.

No olvidemos que la cuota de mercado de Google supera el 90\% en la mayor parte de países de la Unión Europea. Por tanto, estamos ante unas prácticas que difícilmente un particular puede llegar a eliminar y que en todo caso requieren demandas muy costosas al tener que denunciar a la compañía matriz en California (USA). $\mathrm{Si}$ el buscador limitara estas indexaciones y cumpliera el Derecho al Olvido, entonces gran parte del problema desaparecería. Mientras tanto, sólo podemos rogar porque estas indefensiones no continúen destrozando reputaciones online y en algunas ocasiones, hasta vidas.

\section{Referencias}

BÖHM, M., LEIMEISTER, S., RIEDL, C., Y KRCMAR, H. Cloud Computing- Outsourcing 2.0 or a new Business Model for IT Provisioning? Application management (pp. 31-56), 2011, Gabler.

BROWN, J.J. Y REINGEN, P.H. "Social ties and word-of-mouth referral behavior", Journal of Consumer Research, Vol. 14, December, pp. 350-62, 1987.

CHATTERJEE, P. "Online reviews: do consumers use them", in Gilly, M.C. and Meyers-Levy, J. (Eds), Advances in Consumer Research, Vol. 28, Association for Consumer Research, Valdosta, GA, pp. 129-33, 2001.

CHEN, Y. Y XIE, J. "Online consumer review: word-of-mouth as a new element of marketing communication mix", Management Science, Vol. 54 No. 3, pp. 477-91, 2008.

COUTINHO, C. P. A qualidade da investigação educativa de natureza qualitativa: questões relativas à fidelidade e validade. Educação Unisinos, 12(1), 5-15, 2008. 
DELLAROCAS, C. "Immunizing online reputation reporting systems against unfair ratings and discriminatory behaviour", Proceedings of the 2nd ACM Conference on Electronic Commerce, Association for Computing Machinery, Minneapolis, MN, pp. 150-6, 2000

FRANTZIOU E. Further Developments in the Right to be Forgotten: The European Court of Justice's Judgment in Case C131/12, Google Spain, SL, Google Inc v Agencia Espanola de Proteccion de Datos. Human Rights Law Review; 14 (4): 761 777, 2014. doi: http://dx.doi.org/10.1093/hrlr/ngu033

GLASER, B.Y STRAUSS, A. The Discovery of Grounded Theory: Strategies for Qualitative Research. 1967, New York, Aldine. GODES, D. Y MAYZLIN, D. "Using online conversations to study word-of-mouth communication", Marketing Science, Vol. 23 No. 4, pp. 545-60, 2004

GUPTA, P., SEETHARAMAN, A., Y RAJ, J. R. The usage and adoption of cloud computing by small and medium businesses. International Journal of Information Management, 33(5), 861-874, 2013

LIAN, J. W., YEN, D. C., YWANG, Y. T. An exploratory study to understand the critical factors affecting the decision to adopt cloud computing in Taiwan hospital. International Journal of Information Management, 34(1), 28-36, 2014.

MAYER-SCHÖNBERGER, V. Delete: The Virtue of Forgetting in the Digital Age, Princeton and Oxford 2, pp. 1-3, 2009.

MAYOR GÓMEZ, R. Contenido y Novedades del Reglamento General de Protección de Datos de la UE (Reglamento Ue 2016/679, De 27 De Abril De 2016). GABILEX, 6 Junta de Castilla La Mancha. 2016.

MITLIAGA, V. Z. Online privacy: Explaining the nature and special features of the right to seek protection. Journal of Information, Communication and Ethics in Society, 2(3), 159-167, 2004

PALOS, P.R. Y CORREIA, M.B. La actitud de los recursos humanos de las organizaciones ante la complejidad de las aplicaciones SaaS. Dos Algarves: A Multidisciplinary e-Journal, 28, 87-103, 2016a. DOl: http://dx.doi.org/10.18089/DAMeJ.2016.28.6

PALOS, P.R. Y CORREIA, M.B. The Paradigm of the Cloud and Web Accessibility and its Consequences in Europe. Conference DSAI 2016, Vila Real, Portugal. Communications of ACM 978-1-4503-4748-8/16/12, 2016b. DOI: http://dx.doi.org/10.1145/3019943.3019995

PALOS, P. R., Y AGUAYO-CAMACHO, M. Los cambios organizacionales y la nube en el entorno turístico: estudio de caso. International Journal of World of Tourism, Vol. 3, N 5,2016

PALOS-SANCHEZ, P.; SAURA, J.R. The Effect of Internet Searches on Afforestation: The Case of a Green Search Engine. Forests 2018, 9, 51 .

PARK, D.-H., LEE, J. Y HAN, I. "The effect of on-line consumer reviews on consumer purchasing intention: the moderating role of involvement", International Journal of Electronic Commerce, Vol. 11 No. 4, pp. 125-48, 2007.

PUNCH, K. F. Introduction to Social Research. Quantitative \& Qualitative Approaches. London, 1998, Sage Publications.

SAURA, J.R., PALOS, P, DEBASA NAVALPOTRO, F. El problema de la Reputación Online y Motores de Búsqueda: Derecho al Olvido. Cadernos de Dereito Actual 8, pp. 221-229

SILBERLEIB, L. (2016). El Derecho al olvido y la persistencia de la memoria. Información, cultura y sociedad, (35), 125-136, 2017.

STREICH, G W. Is There a Right to Forget? Historical Injustices, Race, Memory, and Identity, New Political Science, Vol. 24/4 pp. 525-542, 2002

SULTAN, N. A. Reaching for the "cloud": How SMEs can manage. International Journal of Information Management, 31(3), 272-278, 2011.

SWIS FEDERAL COURT, BGE 122 III 449, 1996.

SWISS FEDERAL COURT, BGE 70 II 127; to the right of surviving relatives being entitled to make a claim on behalf of the deceased person, see Swiss Federal Court, December 14, 1978, BGE 104 II 225, C. 5b, 1944.

SWISS FEDERAL COURT. 5C.156/2003, 2003.

WEBER, R. H. The Right to Be Forgotten: More Than a Pandora's Box?, 2, 2011. JIPITEC 120.

WERRO, F. Y BELSER, E.M. Le droit à l'oubli et ses limites, medialex 1997, pp. 99 et seq.; for a detailed discussion see Mirjam Teitler, Der rechtskräftig verurteilte Straftäter und seine Persönlichkeitsrechte im Spannungsfeld zwischen öffentlichem Informationsinteresse, Persönlichkeitsschutz und Kommerz, Zurich 2008, pp. 29-98. 
WERRO F. The Right to Inform v. the Right to be Forgotten: A Transatlantic Crash, in: Liability in the Third Millennium, Liber Amicorum Gert Brüggemeier, Baden-Baden, pp. 285 et seq, 2009.

WU, W. W., LAN, L. W., AND LEE, Y. T. Exploring decisive factors affecting an organization's SaaS adoption: A case study. International Journal of Information Management, 31(6), 556-563, 2011.

YOUSEFF, L., BUTRICO, M., AND DA SILVA, D. Toward a unified ontology of cloud computing. In Grid Computing Environments Workshop, 2008. GCE'08 (pp. 1-10). IEEE.

\section{Datos sobre los autores}

\section{Pedro R. Palos-Sanchez}

Doctor en Economía de la Empresa por la Universidad de Sevilla y Profesor Asociado de Investigación de Mercados en el Departamento de Dirección de Empresas y Sociología, impartiendo docencia en la Facultad de Empresa, Finanzas y Turismo de la Universidad de Extremadura. Sus principales intereses de investigación son Economía digital, Emprendimiento y Management, siendo sus líneas de investigación Marketing Digital y Sistemas de Información.

ppalos@unex.es

\section{Rafael Robina Ramírez}

Doctor en Economía por la Universidad de Extremadura y Profesor de Administración de Empresas en el Departamento de Dirección de Empresas y Sociología, impartiendo docencia en la Facultad de Empresa, Finanzas y Turismo de la Universidad de Extremadura. Sus principales intereses de investigación son Ética para los negocios y el turismo.

rrobina@unex.es

\section{Luis Manuel Cerdá Suárez}

Doctor en Economía por la Universidad Autónoma de Madrid. Profesor de Marketing en la Universidad Internacional de La Rioja (España). Su investigación actual se centra en Marketing público y sin fines de lucro, en áreas diversas del marketing y la investigación de mercados, la innovación educativa, el comercio minorista y electrónico, los centros comerciales y la revitalización comercial urbana.

luis.cerda@unir.net

Recibido - Received: 2017-02-14

Aceptado - Accepted: 2018-02-07

\section{(c) $)$ EY}

This work is licensed under a Creative Commons Attribution 4.0

United States License.

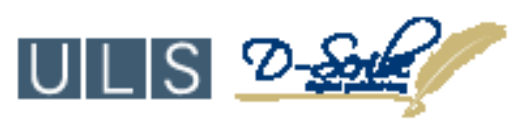

This journal is published by the University Library System of the University of Pittsburgh as part of its D-Scribe Digital Publishing Program and is cosponsored by the University of Pittsburgh Press. 